\title{
Palliative radiotherapy: history, recent advances, and future directions
}

\author{
Stephen T. Lutz \\ Eastern Woods Radiation Oncology, Blanchard Valley Health Organization, Findlay, OH, USA \\ Correspondence to: Stephen T. Lutz, MD, MS, FASTRO. Eastern Woods Radiation Oncology, Blanchard Valley Health Organization, 15990 Medical \\ Drive South, Findlay, OH 45840, USA. Email: sttrlutz@gmail.com.
}

\begin{abstract}
Radiotherapy has been used to palliate cancer symptoms since shortly after the time that $\mathrm{X}$ rays were discovered late in the 1800 's. The $20^{\text {th }}$ century witnessed improvements in treatment planning and delivery that permitted radiotherapy to serve as a successful, timely, and cost-efficient palliative intervention. Palliative radiation oncology has risen to the level of its own subspecialty, as evidenced by the formation of palliative radiation oncology clinical services and dedicated palliative radiotherapy guidelines, while additional changes have begun to alter the very definition and goals of palliative radiotherapy. Local treatment may now be offered with dual goals of symptom relief and the potential for increased disease-free or overall survival. While these new directions show great promise, novel strategies must be formulated to manage the increased complexity, workload, and cost of these approaches.
\end{abstract}

Keywords: Radiotherapy; palliative care; technological advances

Submitted Jan 21, 2019. Accepted for publication Mar 04, 2019.

doi: 10.21037/apm.2019.03.02

View this article at: http://dx.doi.org/10.21037/apm.2019.03.02

\section{Introduction}

The earliest uses of external beam radiotherapy included palliative treatment of bleeding from superficial tumors and pain due to enlarging masses (1). The therapeutic window for palliative radiotherapy was widened with development of the field of radiobiology and the concordant understanding of normal tissue tolerances and dose fractionation (2). The availability of higher energy photons in the $20^{\text {th }}$ century allowed for palliative treatment of deeper-seated tumors could be irradiated (3). Furthermore, advances in highly conformal therapy around the turn of the millennium permitted methods for dose delivery that were not previously conceptualized (4). Still, current forces are changing the very nature of the term 'palliative radiotherapy' in a way that rivals the magnitude of those advances which have taken place in phases since the discovery of radiation by Roentgen in 1895 . Here we explore the ongoing ascent of palliative radiotherapy to a dedicated subspecialty, the merging of palliative and curative intent radiotherapy, and the prospects for improved palliative radiotherapy care moving forward.

\section{Palliative radiotherapy as a subspecialty}

The field of palliative radiotherapy has followed a natural and accelerating progression toward its rightful place as a defined subspecialty in oncology. These advances have evolved to include the formation of dedicated clinical services, the influence of the subspecialty on treatment guidelines, and the creation of an international organization fully devoted to the topic of palliative radiotherapy. The development of dedicated palliative radiation oncology clinical services within academic radiation oncology departments has greatly enhanced the standing of the subspecialty in the oncology community $(5,6)$. These dedicated services permit focused training of palliative care principles that may not otherwise be presented in the average residency training setting (7). The uniformity of care made possible by the existence of a dedicated palliative radiation oncology service permits more robust brainstorming, conceptualization, and implementation of 
palliative radiotherapy research initiatives.

Most cancers increase in incidence with age, so the prevalence of the most common tumor types will also increase with the relative aging of the population of the developed world (8). This population dynamic will require even more dedication to end of life oncology care. Radiation oncology trainees interested in palliative care can seek out dual certification in Hospice and Palliative Medicine (HPM) (9). Candidates for HPM certification may access training through one of a variety of specialties, including a pathway for dual certification in both Radiation Oncology as well as HPM through the American Board of Radiology. Recently, radiation oncologists with dual certification in HPM have increased in number and influence. However, many radiation oncology physicians obtained dual certification prior to the requirement for fellowship training to achieve that status. The American Board of Internal Medicine requirement for a palliative care fellowship to sit for board examination in HPM places a strict limit upon the total number of diplomats in the specialty (10). The current structural organization of radiotherapy residency training programs may serve as a barrier for specialists who wish to seek dual certification. The time spent matriculating in a Hospice and Palliative Care fellowship does not concurrently fulfill the requirements needed to complete a residency in radiation oncology. Whereas numerous training programs permit research time spent in laboratory settings to suffice as legitimate training time in the specialty, the rigorous and highly clinically relevant time spent completing palliative care training does not.

Still, major trends do suggest that the topic of palliative oncology has gained importance on a national level. The development of guidelines with either a fully dedicated or partial emphasis on palliative issues includes those authored by the American Society for Radiation Oncology (ASTRO), American Radium Society (ARS), and the National Comprehensive Cancer Network (NCCN) (11-13). Over the past decade, these national organizations have shown an increasing dedication to creating palliative radiotherapy guidelines. ASTRO has released guideline publications dealing with purely or mostly palliative clinical circumstances such as painful bone metastases, brain metastases, locally advanced lung cancer, and glioblastoma multiforme (14-17). The publication of these guidelines has included collaborative work between specialists and endorsement by other pertinent medical specialty societies.

Furthermore, the Society for Palliative Radiation Oncology (SPRO) was formed to include palliative radiotherapy experts from all over the world who pool their collective efforts to further research, education, and advocacy (18). This group represents practices in countries and locales with widely varying practice patterns, reimbursement models, and resource availability.

\section{The influence of emerging technologies on palliative radiotherapy}

Advances in technology, treatment delivery, and the understanding about tumor behavior have come to change the very definition of palliative radiotherapy. These improvements have blurred the line between palliative and curative intent care, and they have created an entirely new set of benefits and risks for patients with metastatic cancer. The World Health Organization has historically defined palliative care as follows (19):

"Palliative care is an approach that improves the quality of life of patients and their families facing the problem associated with life-threatening illness, through the prevention and relief of suffering by means of early identification and impeccable assessment and treatment of pain and other problems, physical, psychosocial and spiritual."

Therapy may be therefore delivered with the concurrent goals of symptom palliation and cure. Still, most radiotherapy dosing prescriptions require the clinician to delineate between goals of palliative or curative intent. So, 'palliative intent' radiotherapy has commonly implied what may be better described as treatment offered with 'purely palliative intent'. Patients who fall into this category have historically been treated with the most expedient and cost-effective radiotherapy regimens. These treatment courses were best delivered using minimally complex and hypofractionated courses. However, given multiple new reports, palliative radiotherapy may now include concurrent goals of symptoms relief, symptom prevention, local tumor control, and possible cure $(20,21)$. Whereas patterns of care may have previously led radiation oncologists to limit palliative treatment to symptomatic disease sites, they may now recommend therapy to sites that are minimally symptomatic or even symptom-free.

There has been an increasing emphasis on the use of local treatments for patients with metastatic disease with the goal of long-term disease-free survival or even cure. Until recently the very definition of the term 'oligometastatic disease' has been undergoing transitions as treatment trials have been written and completed (22). Two recently-reported studies have shed light on this 
issue. In the first study, NSCLC patients with three or fewer metastases and at least a stable response to initial systemic therapy were randomized to no additional treatment versus radiotherapy to all metastatic sites. Addition of radiotherapy was associated with an increase in disease-free survival from 4.4 to 14.2 months and on overall survival from 17.0 to 41.2 months with the addition of radiotherapy to all metastatic sites (23). Similar findings were found in the SABR-COMET phase II randomized trial, in which patients with a controlled primary site of disease and five or fewer metastases from breast, prostate, lung, or colorectal cancer had improved overall survival (28 to 41 months) with the addition of radiotherapy to all metastatic sites (24).

The increased life expectancy of the patients in this oligometastatic cohort may create a need for greater resources directed at both the cancer treatments, themselves, and to the long-term care of patients with multiple medical problems. For instance, patients who may face cognitive deficits such as short-term memory loss following whole brain radiotherapy may now more commonly live long enough to face the risks associated with that treatment approach $(25,26)$. The very notion that patients may live years rather than months with metastatic disease will cause the specialty of radiation oncology to rethink issues as basic as dose fractionation and long-term side effects. The prolonged side effect risks in patients who are treated with life-prolonging immunotherapeutic agents can create a time frame for toxicity to persist for an indefinite basis. The concept of normal tissue tolerance will need to include these new variables. In the past, patients facing treatment-induced memory deficits 9-12 months following whole brain radiotherapy could still be treated with less fear of late effects if their life expectancy measured only 2-4 months. However, if those same patients now potentially live greater than 1 year due to the benefits of improved systemic therapy, the potential sub-acute and long-term side effect risks of radiotherapy need to be more closely evaluated. Data have shown that novel treatment approaches such as hippocampal sparing during whole brain radiotherapy can lead to a statistically significantly lower rate of memory deficits 6 months after therapy (26). The need for a greater durability of response of palliativeintent radiotherapy increases with life expectancy, as well. So, the transition from measuring pure symptom relief to local control endpoints in patients treated with palliative radiotherapy seems an appropriate subspecialty goal to approach.

\section{Future directions in palliative radiotherapy}

The prospects for improved palliative radiotherapy care include accelerating advances in technological capabilities of radiotherapy calculation and delivery, increasing the ability to evaluate data relevant to palliative care outcomes, and streamlining information transfer and clinical processes relevant to this patient group. Moore's law suggests that computer processing power doubles about every 2 years (27). This logarithmic improvement in computer processing capabilities has provided huge benefits in the provision of radiotherapy. However, the examination and assimilation of technological advances into clinical medicine cannot currently proceed at a similar pace. One must acknowledge that the adoption of the use of multi-leaf collimation rather than poured Cerrobend blocks on linear accelerators was not first exhaustively investigated in prospective trials. The prima facia evidence for the use of the newer technology led to its near uniform use over a relatively short period. Just as one would not advocate for returning to the use of an earlier form of desktop computer or mobile phone, the move to advanced technology in radiotherapy proceeds only in a forward direction. Still, even when trials are completed and suggest newer methods for treatment, there exists a lag in time between documented improvements in patient care and the integration of that information into treatment guidelines and daily practice (28).

Additionally, patient autonomy is one of the main tenets of the healthcare system in the United States. Palliative care on both an individual and systemic level needs to account for the mindset and goals of an individual facing their own death. Individuals may view an expensive, palliative intervention as unreasonable on a societal level but justified in their own case. The definition of futile care therefore depends upon the viewpoint of the person who is discussing the topic. A person who lays dying and seeks hope may envision an intervention with a low response rate to be a worthy undertaking. One can argue that the needs of the many outweigh the needs of the one, but societal pressures to limit care can lead to emotionally charged discussions. Family members of patients commonly act as the impetus for discussions about continuing aggressive care, so they too must be invited to join in discussions regarding care goals. The higher cost associated with complex palliative radiotherapy techniques must be measured while considering the expenses associated with prolonged use of newer systemic agents $(29,30)$. Costs may include the direct expense of treatment, the secondary costs associated with 
managing treatment-related side effects, and the time and money needed for the provision of care by loved ones or dedicated long term care facilities.

Many of the processes of providing palliative radiotherapy have become more streamlined, though increasing complexities of treatment planning, quality assurance, and delivery will require further efforts in efficiency. Multi-slice CT scanners have permitted quicker studies and greater throughput in the CT simulator room than did single slice scanners. Treatment planning software now allows for rapid auto contouring of target and avoidance structures. Dose calculation algorithms require only seconds rather than the minutes, or hours, needed in the past. From a programmatic standpoint, the formation of rapid-response palliative radiotherapy services in some academic centers has permitted a greater ability for patients to undergo assessment, simulation, dose planning, and treatment in the same day $(31,32)$.

However, ongoing impediments to streamlined care include uncertainties regarding the timeliness, and even the very willingness, of third-party payers to 'approve' treatment for these patients. Radiotherapy clinics in a variety of clinical settings could also improve palliative care availability by simply saving consultation and CT simulation time slots for patients that are referred for symptomatic disease. The appropriate quality assurance methods should be streamlined to better permit timely initiation of complex palliative radiotherapy treatments, such as stereotactic body radiation therapy. Evaluation of reporting through the Radiation Oncology and Incident Learning System may continue to provide a focus upon those areas of quality assurance that need additional focus versus other tasks that are less likely to lead to unwanted outcomes (33).

The adoption of data into clinical practice depends upon the penetration of new care patterns into organizational treatment guidelines. Prospective randomized trials require time to formulate, review, accrue, gather adequate follow up, write-up, and publish. Even then, the transition from high level data to guideline formation to adoption by practices may take years. Guidelines are updated, on average, no more quickly than every twelve months, but oftentimes substantially longer. The NCCN guidelines are updated yearly, while updates of the ASTRO guidelines may take place as slowly as every 3 to 4 years (34). As the pace of scientific and technologic advances quicken, the more that the existing methodology for translating those advances into practice patterns seems outdated. To address these needs, some research cooperatives are evaluating options for research based upon a smaller number of data sets accrued over a shorter period. Guideline committees may do well to strive to report updates on a real-time basis as newer data are released. For example, an existing panel of palliative radiation oncology experts could assess data as it is released and determine if the results are sufficiently compelling to create an updated guideline that could be released on line or through an app. Lastly, the adoption of new radiotherapy technologies can only proceed with further collaboration with guidelines committees that evaluate the use of new systemic therapies.

\section{Conclusions}

Palliative radiotherapy has progressed through several phases during the more than 120 years since the first discovery of the $\mathrm{X}$ ray. While forces led to palliative approaches that optimized efficiency and cost effectiveness, newer data suggest that aggressive treatment using highly conformal therapy for patients with limited metastases can both improve symptoms and increase disease-free and overall survival. The future of palliative radiotherapy will require evaluation and management of the increased technological complexity, time commitment, and costs inherent to these new approaches.

\section{Acknowledgments}

I would like to thank Analese Lutz for helping to edit the formatting and content of this manuscript.

\section{Footnote}

Conflicts of Interest: ST Lutz receives royalties from the sale of the textbooks entitled "Handbook of Palliative Radiation Therapy and Radiation Oncology in Palliative Cancer Care”.

\section{References}

1. Freund L. Elements of general radio-therapy for practitioners. Rebman, New York, 1904. (Accessed January 13, 2019). Available online: https://archive.org/details/ elementsgeneral00freugoog/page/n12

2. Coutard H. The results and methods of treatment of cancer by radiation. Ann Surg 1937;106:584-98.

3. Parker RG. Palliative radiotherapy. JAMA 1964;190:1000-2.

4. Shaw E, Scott C, Souhami L, et al. Single dose radiosurgical treatment of recurrent previously irradiated 
primary brain tumors and brain metastases: final report of RTOG protocol 90-05. Int J Radiat Oncol Biol Phys 2000;47:291-8.

5. Garcia M, Balboni T, Braunstein S, et al. Acute pain management in radiation oncology: Quality of care and the impact of an integrated palliative oncology service. J Clin Oncol 2016;34:195.

6. Chang S, Smith C, Morrison R, et al. A palliative radiation oncology consult service's impact on care of advanced cancer patients with symptomatic bone metastases. J Clin Oncol 2015;33:110.

7. Schuster J, Tam K, Skoro N, et al. Influence of palliative care consult on radiation therapy utilization for metastatic cancer patients within the last six months. J Clin Oncol 2014;32:149.

8. White MC, Holman D, Boehm J, et al. Age and Cancer Risk: A Potentially Modifiable Relationship. Am J Prev Med 2014;46:S7-15.

9. Lutz S, Lupu D, Johnstone P, et al. The Influence of the Newly Formed Hospice and Palliative Medicine Subspecialty on Radiation Oncology and End-of-Life Care. J Am Coll Radiol 2008;5:1102-5.

10. Accreditation Council for Graduate Medical Education: ACGME Program Requirements for Graduate Medical Education in Hospice and Palliative Medicine. ACGME approved focused revision: February 4, 2018; effective: July 1, 2018. (Accessed January 13, 2019). Available online: https://www.acgme.org/Portals/0/PFAssets/ProgramR equirements/540HospicePalliativeMedicine1YR2018. pdf?ver=2018-02-16-083923-063

11. American Society for Radiation Oncology: Clinical Guidelines. (Accessed January 13, 2019). Available online: https://www.astro.org/Patient-Care-and-Research/ Clinical-Practice-Statements/Clinical-Practice-Guidelines

12. American Radium Society: Appropriate Use Criteria. (Accessed February 27, 2019). Available online: https:// www.americanradiumsociety.org/page/auc

13. National Comprehensive Cancer Network: Palliative Care. (Accessed February 27, 2019). Available online: https://www.nccn.org/professionals/physician_gls/PDF/ palliative.pdf

14. Lutz S, Balboni T, Jones J, et al. American Society for Radiation Oncology: ASTRO Guideline on Palliative Radiation Therapy for Bone Metastases - Update. Pract Radiat Oncol 2017;7:4-12.

15. Tsao MN, Rades D, Wirth A, et al. American Society for Radiation Oncology: ASTRO Guideline on Radiotherapeutic and Surgical Management for Newly
Diagnosed Brain Metastasis(es). Pract Radiat Oncol 2012;2:210-25.

16. Moeller B, Balagamwala E, Chen A, et al. American Society for Radiation Oncology: ASTRO Guideline on Palliative Radiation Therapy for Non-small Cell Lung Cancer (NSCLC) - Update. Pract Radiat Oncol 2018;8:245-50.

17. Cabrera AR, Kirkpatrick JP, Fiveash JB, et al. Radiation therapy for glioblastoma: Executive summary of an American Society for Radiation Oncology EvidenceBased Clinical Practice Guideline. Pract Radiat Oncol 2016;6:217-25.

18. Wei R, Simone C, Lutz S. Society for palliative radiation oncology: founding, vision, and report from the second annual meeting. Ann Palliat Med 2016;5:74-5.

19. World Health Organization: WHO definition of palliative care. (Accessed January 13, 2019). Available online: https:// www.who.int/cancer/palliative/definition/en/

20. Tree AC, Knoo VS, Eeles RA, et al. Stereotactic body radiotherapy for oligometastases. Lancet Oncol 2013;14:e28-37.

21. Hong JC, Salama JK. The expanding role of stereotactic body radiation therapy in oligometastatic solid tumors: What do we know and where are we going? Cancer Treat Rev 2017;52:22-32.

22. Kissel M, Helou J, Thariat J. Definitions of oligometastatic disease and new treatment concepts. Bull Cancer 2018;105:696-706.

23. Gomez D, Chang C, Zhang J, et al. Local consolidative therapy (LCT) improves overall survival (OS) compared to maintenance therapy/observation in oligometastatic non-small cell lung cancer (NSCLC): final results of a multicenter, randomized controlled phase 2 trial. Int J Radiat Oncol Biol Phys 2018;102:1604.

24. Palma D, Olson R, Harrow S, et al. Stereotactic ablative radiation therapy for the comprehensive treatment of oligometastatic tumors (SABR-COMET): results of a randomized trial. Int J Radiat Oncol Biol Phys 2018;102:S3-4.

25. Margolin K, Ernstoff M, Hamid O, et al. Ipilimumab in patients with melanoma and brain metastases: an openlabel, phase 2 trial. Lancet Oncol 2012;13:459-65.

26. Gondi V, Deshmukh S, Brown PD, et al. Preservation of Neurocognitive Function (NCF) with Conformal Avoidance of the Hippocampus during Whole-Brain Radiotherapy (HA-WBRT) for Brain Metastases: Preliminary Results of Phase III Trial NRG Oncology CC001. Int J Radiat Oncol Biol Phys 2018;102:1607. 
27. Moore G. Cramming more components onto integrated circuits. Electronics 1965;38:114-7.

28. Qunaj L, Jain RH, Atoria CL, et al. Delays in the Publication of Important Clinical Trial Findings in Oncology. JAMA Oncol 2018;4;e180264.

29. Van de Werf E, Verstraete J, Lievens Y. The cost of radiotherapy in a decade of technology evolution. Radiother Oncol 2012;102:148-53.

30. Verma V, Sprave T, Hague W, et al. A systematic review of the cost and cost-effectiveness studies of immune checkpoint inhibitors. J Immunother Cancer 2018;6:128.

31. Danjoux C, Chow E, Drossos A, et al. An innovative rapid response radiotherapy program to reduce waiting

Cite this article as: Lutz ST. Palliative radiotherapy: history, recent advances, and future directions. Ann Palliat Med 2019;8(3):240-245. doi: 10.21037/apm.2019.03.02 time for palliative radiotherapy. Support Care Cancer 2006;14:38-43.

32. Razvi Y, Chan S, Zhang L, et al. A review of the Rapid Response Radiotherapy Program in patients with advanced cancer referred for palliative radiotherapy over two decades. Support Care Cancer 2019;27:2131-34.

33. Hoopes DJ, Dicker AP, Eads NL, et al. RO-ILS: Radiation Oncology Incident Learning System: A report from the first year of experience. Pract Radiat Oncol 2015;5:312-8.

34. National Comprehensive Cancer Network: Development and Update of the NCCN Guidelines ${ }^{\circledR}$. (Accessed January 13, 2019). Available online: https://www.nccn.org/ professionals/development.aspx 